\title{
Desarrollo moral de los estudiantes de un programa de enfermeria
}

\section{Moral development of the students of a nursing program}

\author{
Luz-F Casas-A ${ }^{1}$; Luz-E Ibáñez-A ${ }^{1}$; Dora-I Parra ${ }^{1}$
}

Forma de citar: Casas LF, Ibañez LE, Parra DI. Desarrollo moral de los estudiantes de un programa de enfermería. Rev Univ Ind Santander Salud. 2018; 50(3): 247-256. doi: http://dx.doi.org/10.18273/revsal.v50n3-2018009 (c) (1)

\section{Resumen}

Objetivo: Determinar el nivel de desarrollo moral de los estudiantes del programa de Enfermería de la Universidad Industrial de Santander. Materiales y métodos: Estudio de corte transversal que utilizó el Cuestionario de Opinión sobre Problemas Sociales (COPS) fundamentado en la clasificación propuesta por Kohlberg y reestructurado por Rest. La población blanco correspondió a 187 estudiantes de enfermería de la Universidad Industrial de Santander, matriculados en los núcleos temáticos de cuidado. Resultados: Se pudo establecer el desarrollo de la competencia moral en cada uno de sus niveles: pre convencional, convencional y pos convencional, evidenciándose una correspondencia del 42.9\% para el desarrollo moral convencional, seguida del 39.4\% para el post convencional, y del $9.5 \%$ para el pre convencional. Conclusiones: La mayoría de los estudiantes de enfermería se encontraban en el nivel convencional de su desarrollo moral, aspecto que permite inferir que no existen diferencias entre los niveles académicos, excepto en los estudiantes del tercer nivel, quienes obtuvieron un mayor desarrollo moral post convencional, lo cual puede obedecer a la coincidencia entre la finalización de la asignatura de ética en enfermería, propia del plan curricular, y el momento en que se llevó a cabo la investigación.

Palabras clave: Desarrollo moral; Estudiantes de enfermería; Educación superior.

\begin{abstract}
Objective: To determine the level of moral development of the students of the Nursing program of the Universidad Industrial de Santander. Materials and methods: Cross-sectional study using the Opinion Questionnaire on Social Problems (COPS) based on the classification proposed by Kohlberg and restructured by Rest. The target population corresponded of 187 nursing students from the Universidad Industrial de Santander, enrolled in the thematic nuclei of carefulness. Results: It was possible to establish the development of moral competence in each of its levels: preconventional, conventional and post conventional, evidencing a correspondence of $42.9 \%$ for conventional moral development, followed by $39.4 \%$ for the conventional post, and $9.5 \%$ for the pre-conventional. Conclusions: The majority of nursing students were at the conventional level of moral development, aspect that allows to infer that there are no differences between academic levels. Except in the third level students, whom obtained a greater postconventional moral development, which can obey of the coincidence between the completion of the subject of ethics in nursing, contained in the curricular plan, and the time when the research was carried out.
\end{abstract}

Keywords: Moral development; Nursing students; High education.

1. Universidad Industrial de Santander. Bucaramanga, Colombia.

Correspondencia: Luz F Casas. Dirección: Carrera 32 29-31, Bucaramanga. Teléfono: +57 7634 5745. Correo electrónico: luzfcasas@uis.edu.co. 


\section{Introducción}

El primer llamado que hace la bioética frente a la vida, es su conocimiento, cuidado y posibilitamiento en sus niveles individual, colectivo y global ${ }^{1}$. Este llamado implica el desarrollo de un profundo sentido de responsabilidad con la vida, aspecto que coincide, en parte, con la idea de Potter, para quien la finalidad del conocimiento es el bien social, razón por la que se requiere de la adopción de prácticas sustentadas en una eticidad que favorezca la construcción de una sabiduría que articule un sentido de responsabilidad y humildad a la ciencia y la tecnología ${ }^{2}$.

La sabiduría en términos de García, el ethos, se adquiere progresivamente en el ámbito de una cultura, gracias a la acogida y ejercicio de virtudes entendidas como costumbres consideradas deseables, las cuales pueden irse adoptando e interiorizando progresivamente acorde a los "más profundos anhelos, ideas personales y sociales, e incluso con las necesidades compartidas para vivir mejor"’. En este sentido, según lo refiere Linde, haciendo referencia a la teoría de desarrollo moral de Kolhberg ${ }^{4}$, la formación de la personalidad moral es un aspecto muy complejo que involucra, tanto procesos cognitivos y evolutivos como "aspectos emocionales, afectivos y biográficos de los sujetos", lo que implica una gran carga "motivacional para progresar en ética y la educación moral", aspecto que debe tenerse en cuenta en el fomento del desarrollo moral, de manera especial en el marco de la educación superior.

En consecuencia, para cualquier programa universitario debe ser un propósito central formar integralmente en el ejercicio profesional y en la promoción de una sabiduría coherente con el desarrollo de una competencia ética y moral frente al cuidado, objetivo medular de la profesión de enfermería y de sus programas curriculares. Es necesario, como lo indica Paravic, "planificar muy bien la formación de la enfermera, con énfasis en los valores de responsabilidad, solidaridad y humanización, lo que significa otorgar dignidad al hombre y sentido a la sociedad". Estos procesos de enseñanza aprendizaje son posibles en la formación por competencias, la cual para Arbeláez, et al. exige ofrecer espacios para que el estudiante, como protagonista de su propio proceso educativo, "imagine, diseñe y construya un proyecto de vida basado en el conocimiento transformador del saber, del hacer, del ser y del convivir"6. En el ámbito del ser, la formación por competencias pretende el desarrollo y fortalecimiento de una habilidad ética, que se hace explícita y se expresa de manera práctica en el contexto de la moral.
Kohlberg concibe que el desarrollo de la competencia moral se establece en un proceso consecutivo y progresivo $^{7} \mathrm{y}$, a partir de su estudio sobre el desarrollo del juicio y carácter moral en jóvenes de diferentes culturas, formula una teoría según la cual el juicio moral se estructura de manera evolutiva en seis estadios, organizándolos de dos en dos, en tres niveles de desarrollo moral: pre convencional (estadios 1 y 2), convencional (estadios 3 y 4 ) y post convencional (estadios 5 y 6). El nivel pre convencional se caracteriza por una moralidad heterónoma e instrumental individualista. Aquí, mientras en el estadio 1 la orientación de la acción se expresa "según castigo y obediencia", en el estadio 2 , la orientación es "relativista instrumental", y la relación con el otro se establece en términos de un intercambio y reciprocidad instrumental.

El nivel convencional, se caracteriza por la adhesión a las normas enmarcadas en relaciones de mutualidad, ley y orden. En este nivel, en el estadio 3, el referente de acción son los estereotipos de "conducta 'natural' o mayoritaria", donde identifica como "buena conducta aquella que agrada o ayuda a los otros y es apropiada para el individuo que la ejerce", cuya intención es la búsqueda de la aprobación externa. Por su parte, en el estadio 4, la orientación está dada "según la ley y el orden", que califica como justa la conducta orientada hacia "la autoridad, las reglas fijas y el mantenimiento del orden social".

En el nivel post convencional la moralidad se expresa en términos de derechos, contrato social y principios éticos universales. Aquí el estadio 5 se distingue por su "orientación legalista del contrato social", aunque la ley no es inamovible y puede ajustarse según "consideraciones racionales de utilidad social". Lo justo, enfocado por valores y opiniones personales, está determinado por "los derechos individuales generales y pautas críticamente examinadas y acordadas por toda la sociedad". Finalmente, el estadio 6 se caracteriza por una "orientación según el principio ético universal". Los principios abstractos y éticos "de justicia, de reciprocidad e igualdad de los derechos humanos, y del respeto por la dignidad de los seres humanos como personas individuales", orientan la decisión de conciencia.

Por su parte, Narváez ${ }^{8}$, y demás autores neokohlbergianos liderados por Rest, adaptaron la teoría de Kohlberg utilizando el término "esquemas" en vez de etapas o estadios, y postularon "tres estructuras en el desarrollo del pensamiento moral" así: "el esquema de intereses primarios", coincidente con los estadios 
2 y 3 de Kohlberg; "el esquema de mantenimiento de normas [...], derivado de la cuarta etapa de Kohlberg; y el esquema posconvencional [...], derivado de las etapas 5 y 6 de Kohlberg". Acorde a esta adaptación, fue diseñado el Defining Issues Test (D.I.T) en el que se descarta la medición del estadio 1 de la entrevista de Kohlberg, dada la incapacidad para responder el instrumento de los infantes en edades muy tempranas que apenas alcanzan este nivel de desarrollo moral. Otro cambio incorporado por Rest, es la reestructuración del estadio 5 en el 5A y 5B propios del esquema postconvencional, según lo cual, en el estadio $5 \mathrm{~A}$ "el sujeto apoya su juicio moral en un legítimo contrato social. La conducta correcta está en función de unos criterios y derechos individuales de carácter general que han sido examinados críticamente y aceptados por la sociedad a la que el sujeto pertenece", mientras que el estadio 5B "se corresponde con un tipo de juicio moral que se caracteriza por ser de tipo intuitivo individualista y humanista" $"$.

Uno de los propósitos centrales de la Escuela de Enfermería de la Universidad Industrial de Santander, es la formación de sus estudiantes en el "cuidado integral de sí mismo, las personas, las familias y los colectivos" ${ }^{\prime \prime}$, aspecto que se trabaja en los diferentes espacios formativos del plan de estudios, mediante un modelo pedagógico de formación por competencias en el que se integran el saber, el ser y el hacer, buscando con ello articular el saber y la praxis de Enfermería con una sabiduría ética de responsabilidad y respeto frente al cuidado. Fundamentados en esta perspectiva, desde el año 2005 a la fecha, se implementó en el plan curricular un componente ético y bioético como eje transcurricular, así como la asignatura de ética en enfermería propia del segundo nivel de formación.

\section{Objetivo}

En este contexto, se planteó como objetivo central determinar el nivel de desarrollo moral de los estudiantes del programa de Enfermería de la Universidad Industrial de Santander, según la categorización de desarrollo moral propuesta de Kohlberg y autores Neokohlbergianos.

\section{Metodología}

Estudio de corte transversal analítico, con una población blanco de 187 estudiantes de enfermería de la Universidad Industrial de Santander, matriculados en los núcleos temáticos de cuidado. Los estudiantes participantes en la investigación fueron los matriculados en los niveles III, V, VII y IX del primer semestre académico del 2016.

Se utilizó el Cuestionario de Opinión sobre Problemas Sociales (COPS), versión corta mexicana del Test de Competencia Moral de Rest, Defining Issues Test (DIT) ${ }^{11}$, el cual fue ajustado, validado e implementado en Colombia por Pérez y Dussán ${ }^{12}$, de quienes se obtuvo el permiso pertinente para su utilización.

El COPS (DIT), además de establecer el desarrollo de la competencia moral de los participantes en los estadios 2, 3, 4, 5A, 5B y 6, permite definir un puntaje $\mathrm{A}$, un puntaje $\mathrm{M}$ y el índice $\mathrm{P} \%$. El puntaje $\mathrm{A}$ determina el pensamiento relativista del encuestado que denota una transición del estadio 4 al 5A; el M es un ítem que no tiene significado y determina la comprensión de los sujetos de las indicaciones para responder el cuestionario. El índice P\% corresponde a la sumatoria de los resultados obtenidos en los estadios 5A, 5B y 6 e indica un índice de pensamiento post convencional.

Este cuestionario parte de tres historias: "Armando y la medicina", "el periódico escolar" y "el dilema del médico". En cada historia se presenta un dilema sociomoral indicando dos valores que orientan soluciones contradictorias frente a la necesidad de tomar una decisión, y se despliegan a partir de cada una tres secciones para ser respondidas consecutivamente. En la primera sección, el participante debe expresar su opinión frente a lo que debe hacer el personaje que se encuentra en dilema en cada historia, en términos de "lo debe hacer", "no lo debe hacer" o "está indeciso". En la segunda sección, se plantean doce enunciados a tener en cuenta en el análisis de los dilemas de cada historia, los cuales deben ser calificados uno a uno en una escala de Likert, según el grado de importancia, de muchísima a ninguna. En la tercera sección el participante debe elegir los cuatro enunciados calificados como de mayor importancia en la segunda sección, y organizarlos de mayor a menor grado de jerarquía.

\section{Procedimiento para la aplicación}

Se hizo invitación pública a los participantes, quienes aceptaron mediante consentimiento informado verbal, fueron convocados a una sala de informática donde se explicaron los procedimientos del estudio y la forma de diligenciar el instrumento dispuesto online. 


\section{Análisis estadístico}

Cada una de las encuestas diligenciadas en la plataforma virtual fue sometida a un proceso de validación mediante un chequeo de inconsistencias en tres procedimientos. Con el primero se descartaron las encuestas que presentaban más de nueve enunciados estimados iguales en la segunda sección de cada historia. El segundo se hizo para verificar la coherencia entre las respuestas de la segunda sección y la jerarquización de la tercera; la validez en este momento se estableció con el chequeo de los dos primeros niveles de jerarquía de la tercera sección, en los que debían ubicarse los enunciados de la segunda sección estimados como de mayor importancia; así, en el primer nivel de jerarquía debía ubicarse el ítem de mayor importancia de la segunda sección, en el segundo nivel debía situarse el segundo ítem estimado más importante de la segunda sección y así hasta llegar al cuarto nivel de jerarquía. Se eliminaron las encuestas que presentaron en cualquier historia más de ocho inconsistencias. En el tercer chequeo se eliminaron los cuestionarios que presentaron al menos una inconsistencia en las tres historias.

Otro proceso de validación de las encuestas fue el chequeo del puntaje $\mathrm{M}$, el cual se estableció a partir de la revisión de la tercera sección de cada historia. Este puntaje se encuentra expreso en uno o dos de los doce enunciados de la segunda sección del cuestionario. Así, si un enunciado correspondiente al puntaje $\mathrm{M}$ estaba ubicado en el primer nivel de jerarquía, se le asignaba un valor de 4 , si se colocaba en el segundo nivel, se le fijaba un valor de 3 , en el tercer nivel se le asignaba un valor de 2 y en el cuarto nivel un valor de 1 . Se descartaron los cuestionarios que alcanzaron una sumatoria del puntaje $M$ superior a 4 , en las tres historias.

Con el total de la muestra validada se efectuó el análisis estadístico según el cual se determinó la distribución de las características sociodemográficas de la población, las variables cualitativas presentadas mediante porcentajes, las medias aritméticas de los estadios del juicio moral del 2 al 6 y del índice P, A y $\mathrm{M}$, por sexo y semestre académico. Se ejecutó la prueba t student (ttest) para la prueba de hipótesis de igualdad de medias en los puntajes de los estadios por sexo, actividad religiosa, social, por edad reagrupada en las categorías de estudiantes $<=20$ años y $>20$ años, por semestre académico en las categorías de tercer semestre y avanzados (todos los demás niveles) y nivel educativo del padre y de la madre reagrupado en básico (primaria y bachillerato) y universitario (pregrado y posgrado). Por último, se analizó la varianza de los puntajes obtenidos en cada estadio por los grupos de semestres académicos, estado laboral del padre y de la madre reagrupada por el tipo de trabajo (1 "Trabajador dependiente", 2 "Trabajador independiente e informal", 3 "Desempleado - pensionado", 4 Otro); todas las pruebas contemplaron un alfa igual a 0.05 .

Se utilizó una prueba Tukey -Kramer a un nivel alfa de 0.05 , cuyo punto crítico se calculó a partir del número de niveles según la variable en comparación y los grados de libertad asociados con el (degrees of freedom) df-residual del ANOVA.

Para determinar la normalidad de las variables se usó la prueba de shapiro wilk a un nivel de 0,05 .

\section{Consideraciones éticas}

Esta investigación contó con el aval de un comité de ética en investigación científica otorgado mediante acta $\mathrm{N}^{\circ} 30$ del 5 diciembre 2014, así como con el consentimiento verbal de los participantes. El anonimato de la información recabada, fue garantizado mediante códigos numéricos.

\section{Resultados}

\section{Características de la población de estudio}

Del total de la población objeto de estudio (187 estudiantes), 147 atendieron la invitación a participar, a quienes se aplicó el COPS (12); sin embargo, la muestra activa fue de 116 cuestionarios, luego de la validación.

Con relación a las características sociodemográficas de la muestra, el rango de edad 19 a 25 años fue el más frecuente con un $87 \%(\mathrm{n}=101)$, debido a que gran parte de los participantes eran de semestres avanzados; el $87 \% \quad(n=101)$ de los estudiantes profesaban una práctica religiosa, el $60 \%(\mathrm{n}=70)$ no participaban activamente en grupos sociales y el $78 \%$ $(\mathrm{n}=91)$ pertenecía al sexo femenino. En cuanto al nivel académico el $47 \%(n=55)$ pertenecían al III nivel, el $30 \%(\mathrm{n}=35)$ al VII nivel, el $17 \%(\mathrm{n}=20)$ al V nivel y solo el $5 \%(\mathrm{n}=6)$ al IX nivel.

\section{Desarrollo del Juicio Moral de los estudiantes}

En relación con los puntajes obtenidos en cada uno de los estadios del desarrollo del juicio moral, se observó un mayor promedio de calificaciones en el estadio $4(26.7 \%)$, seguido del 5A (18.5\%) y del $3(16.2 \%)$. 
Además, se encontró que el promedio de los índices A $(3.7 \pm 0.5)$ y $\mathrm{M}(4.7 \pm 0.5)$ se mantuvo por debajo del $5 \%$ y el índice $\mathrm{P}(39.1 \pm 1.5)$ no superó el valor de $40 \%$.

Frente a los estadios de desarrollo moral por los diferentes grupos de semestres académicos no se encontraron diferencias estadísticamente significativas (Tabla 1 Medias por estadio y del índice P para la muestra, por semestre); sin embargo, al analizar los promedios de los diferentes estadios de desarrollo moral a través de una prueba t student, se encontró evidencia estadísticamente significativa para la diferencia entre el promedio del estadio 5A del grupo de tercer semestre en relación con los demás semestres, como se aprecia en la Tabla 2 Juicio moral tercer semestre versus los demás semestres.

Tabla 1. Medias por estadio y del índice P para la muestra, por semestre.

\begin{tabular}{cccccc}
\hline \multicolumn{7}{c}{ Estadio } & $\begin{array}{c}\mathbf{I I I} \\
\mathbf{n = 5 5} \\
\text { Promedio (DE) }\end{array}$ & $\begin{array}{c}\mathbf{V} \\
\mathbf{n = 2 0} \\
\text { Promedio (DE) }\end{array}$ & $\begin{array}{c}\mathbf{V I I} \\
\mathbf{n = 3 5} \\
\text { Promedio (DE) }\end{array}$ & $\begin{array}{c}\mathbf{I X} \\
\mathbf{n = 6} \\
\text { Promedio (DE) }\end{array}$ & Valor $\mathbf{p}^{*}$ \\
\hline 2 & $8.6(7.6)$ & $9.5(6.7)$ & $10.8(9.6)$ & $9.9(7.30)$ & 0.67 \\
3 & $17.9(10.7)$ & $12.2(13.1)$ & $15.0(9.3)$ & $20.5(11.4)$ & 0.14 \\
4 & $24.1(12.9)$ & $32.8(14.8)$ & $26.3(13.3)$ & $24.5(11.6)$ & 0.09 \\
$5 \mathrm{~A}$ & $21.3(12.5)$ & $15.7(10.2)$ & $16.9(10.3)$ & $13.3(9.2)$ & 0.09 \\
$5 \mathrm{~B}$ & $11.3(7.7)$ & $14.5(8.1)$ & $12.5(9.7)$ & $13.3(7.4)$ & 0.49 \\
6 & $8.2(7.0)$ & $9.2(6.3)$ & $9.2(5.4)$ & $7.7(5.0)$ & 0.84 \\
Índice P & $39.8(18.7)$ & $39.3(14.9)$ & $38.6(13.8)$ & $35(13.1)$ & 0.91 \\
Índice A & $4.5(6.2)$ & $1.2(2.2)$ & $4.1(5.9)$ & $3.3(5.2)$ & 0.15 \\
Índice M & $4.1(4.9)$ & $4.9(4.7)$ & $5.1(5.3)$ & $6.7(5.1)$ & 0.54 \\
\hline
\end{tabular}

DE Desviación Estándar; $\mathrm{n}$ frecuencia absoluta; * ANOVA test.

Fuente: Autores.

Tabla 2. Juicio moral tercer semestre versus los demás semestres.

\begin{tabular}{cccc}
\hline \multicolumn{3}{c}{ Semestre } \\
\hline Estadio & Tercer Promedio (DE) & Avanzado* Promedio (DE) $^{*}$ & Valor $^{* * *}$ \\
\hline 2 & $8.6(7.6)$ & $10.3(1.1)$ & 0.27 \\
3 & $17.9(10.7)$ & $14.6(1.4)$ & 0.10 \\
4 & $24.1(12.9)$ & $28.2(1.8)$ & 0.10 \\
$5 \mathrm{~A}$ & $21.3(12.5)$ & $16.1(1.3)$ & $0.02^{*}$ \\
$5 \mathrm{~B}$ & $11.3(7.7)$ & $13.3(1.1)$ & 0.20 \\
6 & $8.2(7.0)$ & $9.1(0.7)$ & 0.46 \\
Índice P & $39.8(18.7)$ & $38.5(1.8)$ & 0.66 \\
Índice A & $4.5(6.2)$ & $3.1(5.1)$ & 0.18 \\
Índice M & $4.1(4.9)$ & $5.2(5.1)$ & 0.20 \\
\hline
\end{tabular}

DE Desviación Estándar * Avanzado (Quinto, séptimo y noveno semestre). ** Prueba t de Student, ¥ significativo.

Fuente: Autores.

Al analizar la variable sexo se encontró que las puntuaciones de los diferentes estadios en la muestra general crecen del estadio 2 hacia el 4, desde donde se observa una tendencia decreciente en los promedios de las puntuaciones, sin diferencias estadísticamente significativas en los diferentes puntajes de estadios e índice P (Tabla 3. Medias por estadio y del índice P para la muestra, por sexo y edad). Con respecto a la edad, se encontró evidencia estadísticamente significativa para el promedio del grupo menores de 20 años con relación a los demás grupos de edad para el promedio del estadio $5 \mathrm{~A}$, encontrándose valores limítrofes para la significancia estadística en la diferencia entre los promedios del índice M. (Tabla 3). 
Tabla 3. Juicio moral por estadio e índices $\mathrm{P}$, A y M para la muestra, por sexo y edad.

\begin{tabular}{cccccccc}
\hline Estadio & $\begin{array}{c}\text { Promedio } \\
(\mathbf{D E})\end{array}$ & $\begin{array}{c}\text { Hombres } \\
\text { Promedio } \\
(\mathbf{D E})\end{array}$ & $\begin{array}{c}\text { Mujeres } \\
\text { Promedio } \\
(\mathbf{D E})\end{array}$ & $\begin{array}{c}\text { Valor } \\
\mathbf{p}^{*}\end{array}$ & $\begin{array}{c}<=\mathbf{2 0} \\
\text { Promedio } \\
(\mathbf{D E})\end{array}$ & $\begin{array}{c}>\mathbf{2 0} \\
\text { Promedio } \\
(\mathbf{D E})\end{array}$ & $\begin{array}{c}\text { Valor } \\
\mathbf{p}^{*}\end{array}$ \\
\hline 2 & $9.5(8.1)$ & $11.2(9.4)$ & $9.0(7.6)$ & 0.23 & $9.1(7.8)$ & $9.9(8.3)$ & 0.55 \\
3 & $16.2(10.9)$ & $18.4(12.0)$ & $15.6(10.6)$ & 0.25 & $16.9(11.1)$ & $15.3(10.7)$ & 0.42 \\
4 & $26.7(13.5)$ & $23.7(12.0)$ & $26.9(13.8)$ & 0.29 & $25.5(14.1)$ & $27.3(12.6)$ & 0.49 \\
5A & $18.5(11.5)$ & $20.6(9.8)$ & $17.9(11.9)$ & 0.30 & $20.5(13.1)$ & $16(8.5)$ & $0.03^{*}$ \\
5B & $12.3(8.4)$ & $11.3(8.3)$ & $12.5(8.5)$ & 0.50 & $12.4(8.4)$ & $12.3(8.4)$ & 0.94 \\
6 & $8.6(6.3)$ & $7.9(8.3)$ & $8.9(5.7)$ & 0.49 & $8.0(6.9)$ & $9.5(5.3)$ & 0.23 \\
Índice P & $39.1(16.3)$ & $39.8(17.4)$ & $39.3(16.1)$ & 0.79 & $40.9(18.2)$ & $36.73(13.2)$ & 0.17 \\
Índice A & $3.7(0.5)$ & $2.5(0.9)$ & $4.1(0.6)$ & 0.23 & $3.6(5.6)$ & $3.8(5.7)$ & 0.82 \\
Índice M & $4.7(0.5)$ & $4.1(1.1)$ & $4.8(0.5)$ & 0.54 & $3.9(4.9)$ & $5.7(4.9)$ & $0.05^{¥}$ \\
\hline
\end{tabular}

DE Desviación Estándar * Prueba t de Student. ¥ Significativo.

Fuente: Autores.

En la Figura 1. se aprecia como el valor promedio de los puntajes $\mathrm{P}$ en los diferentes semestres es el mismo, también se observa que en los semestres superiores los puntajes no se distribuyeron homogéneamente.

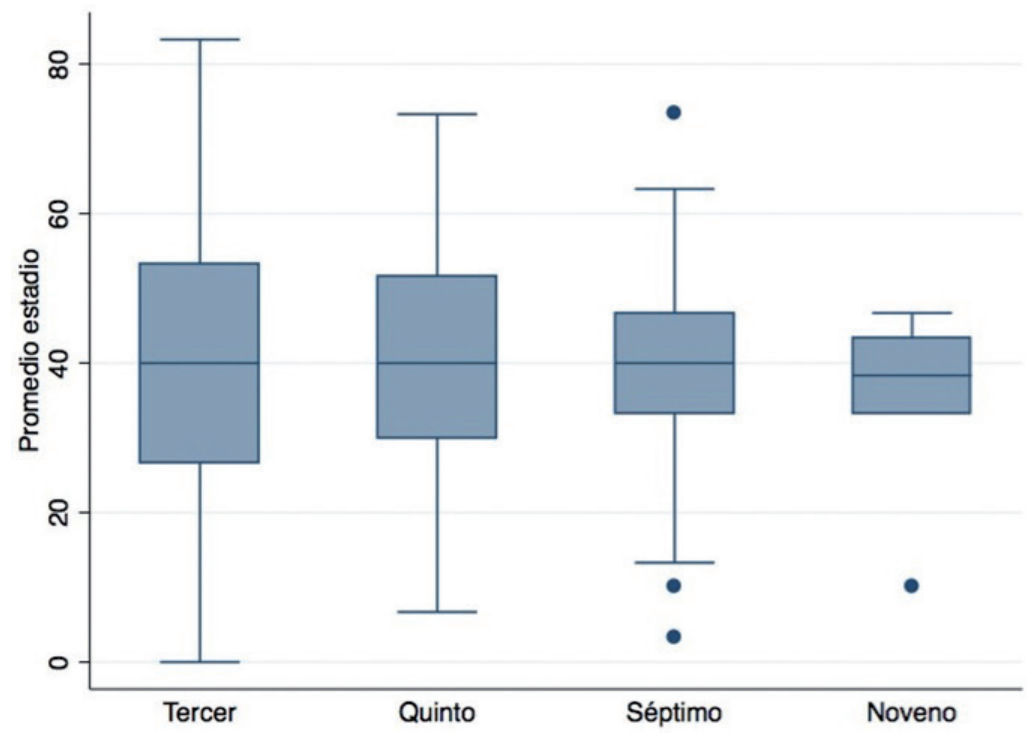

Figura 1. Puntaje P por semestres.

Al analizar los estadios de juicio moral e índices según la práctica religiosa de los estudiantes, se encontró significancia estadística en el índice A, tal como se aprecia en la Tabla 4, pero no con las actividades sociales.

Al comparar los promedios de los diferentes estadios por el nivel de escolaridad del padre y de la madre, como una variable dicotómica con un nivel básico que corresponde a la básica primaria y bachillerato vs universitario que agrupa estudios de pregrado y 252 posgrado, se encontraron diferencias estadísticamente significativas para el estadio $6 \mathrm{p}=0.03$ en el padre y para la madre $\mathrm{p}=0.02$. (Tabla 5).

Al compararse los Estadios de Juicio Moral e índices según el estado laboral del padre del estudiante se encontró evidencia estadísticamente significativa en el índice $\mathrm{M}$ con un puntaje $\mathrm{p}=0.04$. En relación con la madre no se evidenció diferencias estadísticamente significativas en ningún estadio ni índice. Así mismo, al comparar las diferencias entre los promedios del Índice 
M entre los grupos de trabajo del padre, tras realizar la prueba Tukey teniendo en cuenta un punto crítico de 3.6910 para 4 grados de libertad, no se observaron diferencias estadísticamente significativas entre los promedios de ninguno de los grupos.

Tabla 4. Estadios jiucio moral e índices según prácticas religiosas.

\begin{tabular}{ccccccc}
\hline Estadio & \multicolumn{3}{c}{ Práctica religiosa } & \multicolumn{3}{c}{ Actividades sociales } \\
\cline { 2 - 7 } & $\begin{array}{c}\text { NO } \\
\text { Promedio } \\
(\mathbf{D E})\end{array}$ & $\begin{array}{c}\text { SI } \\
\text { Promedio } \\
(\mathbf{D E})\end{array}$ & Valor $\mathbf{p}^{*}$ & $\begin{array}{c}\text { NO } \\
\text { Promedio } \\
(\mathbf{D E})\end{array}$ & $\begin{array}{c}\text { SI Promedio } \\
\text { (DE) }\end{array}$ & Valor p $^{*}$ \\
\hline 2 & $8.9(9.1)$ & $9.5(7.9)$ & 0.76 & $10.0(8.3)$ & $8.5(7.3)$ & 0.31 \\
6 & $16.9(12.7)$ & $16.1(10.7)$ & 0.79 & $15.5(9.6)$ & $17.3(12.7)$ & 0.38 \\
4 & $23.8(8.9)$ & $26.6(14.1)$ & 0.45 & $26.5(13.6)$ & $25.9(13.4)$ & 0.79 \\
$5 \mathrm{~A}$ & $17.8(7.1)$ & $18.7(12.1)$ & 0.78 & $18.3(11.2)$ & $18.9(12.1)$ & 0.75 \\
$5 \mathrm{~B}$ & $11.3(9.2)$ & $12.5(8.3)$ & 0.63 & $11.3(8.6)$ & $12.9(8.1)$ & 0.50 \\
6 & $7.8(6.8)$ & $8.7(6.26)$ & 0.57 & $8.6(6.4)$ & $8.7(6.2)$ & 0.95 \\
Índice P & $36.9(15.6)$ & $39.4(16.5)$ & 0.58 & $38.1(16.7)$ & $40.7(15.8)$ & 0.41 \\
Índice A & $6.9(6.5)$ & $3.2(5.4)$ & $0.02^{*}$ & $3.9(5.3)$ & $3.5(6.1)$ & 0.78 \\
Índice M & $6.7(5.3)$ & $4.4(4.8)$ & 0.09 & $5.1(5.1)$ & $4.0(4.8)$ & 0.27 \\
\hline
\end{tabular}

DE Desviación Estándar *Prueba t de Student, ¥ significativo.

Fuente: Autoras.

Tabla 5. Estadios juicio moral e índices según nivel de educación del padre y la madre.

\begin{tabular}{|c|c|c|c|c|c|c|}
\hline \multirow[b]{2}{*}{ Estadio } & \multicolumn{3}{|c|}{$\begin{array}{c}\begin{array}{c}\text { Nivel educativo del padre } \\
\mathrm{n}=110\end{array} \\
\end{array}$} & \multicolumn{3}{|c|}{$\begin{array}{c}\text { Nivel educativo de la madre } \\
n=116\end{array}$} \\
\hline & $\begin{array}{c}\text { Básico* } \\
\text { n=90 } \\
\text { Promedio (DE) }\end{array}$ & $\begin{array}{c}\text { Universitario } \\
\text { n=20 } \\
\text { Promedio (DE) }\end{array}$ & $\begin{array}{c}\text { Valor } \\
\mathbf{p}^{* *}\end{array}$ & $\begin{array}{c}\text { Básico* } \\
\text { n=85 } \\
\text { Promedio (DE) }\end{array}$ & $\begin{array}{c}\text { Universitario } \\
\text { n=31 } \\
\text { Promedio (DE) }\end{array}$ & $\begin{array}{c}\text { Valor } \\
\mathbf{p}^{* *}\end{array}$ \\
\hline 2 & $9.6(8.5)$ & $8.5(6.4)$ & 0.58 & $9.6(8.5)$ & $9.0(6.7)$ & 0.71 \\
\hline 3 & $16.2(10.9)$ & $15.8(10.6)$ & 0.87 & $16.7(11.0)$ & $14.7(10.7)$ & 0.38 \\
\hline 4 & $27.7(13.5)$ & $22.8(13.1)$ & 0.14 & $26.5(13.2)$ & $25.7(14.4)$ & 0.78 \\
\hline $5 \mathrm{~A}$ & $18.7(11.5)$ & $17.9(12.9)$ & 0.79 & $19.1(11.1)$ & $16.9(12.4)$ & 0.37 \\
\hline $5 \mathrm{~B}$ & $11.7(7.8)$ & $15.2(9.9)$ & 0.08 & $11.7(8.1)$ & $13.9(8.9)$ & 0.20 \\
\hline 6 & $7.9(6.2)$ & $11.2(6.2)$ & $0.03^{¥}$ & $7.8(6.4)$ & $10.9(5.2)$ & $0.02^{*}$ \\
\hline Índice P & $37.8(16.5)$ & $44.3(14.1)$ & 0.10 & $38.0(15.7)$ & $41.9(17.9)$ & 0.26 \\
\hline Índice A & $3.3(5.3)$ & $4.3(6.2)$ & 0.46 & $3.7(5.7)$ & $3.8(5.5)$ & 0.98 \\
\hline Índice M & $4.7(4.9)$ & $3.9(5.1)$ & 0.57 & $4.6(4.9)$ & $4.8(5.1)$ & 0.84 \\
\hline
\end{tabular}

DE Desviación Estandar; *Primaria y Secundaria **Prueba t de Student, ¥ significativo.

Fuente: autoras.

\section{Discusión}

Como se observa en los resultados obtenidos, la mayoría de los participantes del estudio, se encontraban en un desarrollo del juicio moral ubicado en el estadio 4 del nivel convencional, lo que significa según la perspectiva de Kohlberg, que los estudiantes tienen un sistema social y de conciencia motivado por cumplir el propio deber aceptado y dar sostén a las leyes. Aspecto que es repetitivo en los diferentes estudios realizados en estudiantes de educación superior ${ }^{13}$. Se evidencian puntuaciones que no representan significancia estadística en los niveles de competencia del juicio moral, que también han sido detectados en otros estudios donde se ha analizado el mismo fenómeno en estudiantes de enfermería ${ }^{14}$, del mismo modo, se confirma en nuestro estudio, que no hay una influencia directa entre un alto nivel académico del estudiante y el crecimiento moral en general y específicamente del pos convencional dentro del ámbito universitario, 
a diferencia de otros estudios realizados en el ámbito escolar (bachillerato) donde se relata que a mayor edad/ escolaridad hay mayor avance en la moralidad ${ }^{15}$.

Por otra parte, llama la atención el desarrollo moral en el estadio 5A, el cual fue estadísticamente significativo para los estudiantes de tercer nivel del Programa de Enfermería. Asimismo la relación entre el desarrollo moral y la edad, específicamente en el grupo de menores de 20 años. Este hallazgo puede ser explicado dado que el programa de enfermería cuenta con una asignatura específica en ética, la cual se desarrolla durante tres horas semanales en el segundo semestre académico, semestre que acababan de cursar la mayoría de los estudiantes menores de 20 años, condiciones en las que no se encuentran los semestres superiores, pues, el componente ético se aborda con menos intensidad y desde un enfoque aplicado, se puede percibir una deficiente integración entre el desarrollo teórico de la ética y demás componentes teóricos y prácticos del currículo; sin embargo, esto puede ser explicado según Barba y Romo ${ }^{11}$, citando a Rest por las características curriculares y la calidad de las vivencias, así como por las motivaciones y actividades del estudiante de tercer nivel. De igual forma, los resultados relacionados con el rango de edad y el nivel académico como lo indica Perry ${ }^{16}$ con fundamento en Levy (1988), los adolescentes utilizan una variedad de modelos para tomar decisiones morales que les permite formarse en un sistema de valores, código de conducta propia de su etapa y les favorece un comportamiento acorde al sistema de valores y el código de conducta, lo que hace que razonen moralmente acerca de los problemas y del valor de forma temprana.

Con respecto a los índices de los estadios de desarrollo moral, se encontró significancia estadística con las prácticas religiosas, lo cual puede explicarse por la marcada influencia religiosa que la enfermería ha tenido desde sus comienzos, y que en Colombia dicha práctica está arraigada culturalmente y en gran parte de las instituciones de educación básica, la educación religiosa hace parte de los planes de estudio. Estos hechos hacen que el estudiante universitario consolide dicha formación y lo exteriorice, no solo profesando su fe sino desarrollando su sentido crítico racional, que junto a una buena base de principios y valores confirmen la influencia que existe de la religión en el desarrollo moral ${ }^{17}$.

En cuanto a la educación de los padres y el desarrollo moral de los estudiantes, se encontró una relación estadísticamente significativa para el estadio 6; esto sugiere que una mayor educación de los padres favorece que los estudiantes puedan asumir un criterio moral propio ${ }^{18}$.

Al comparar los resultados de este estudio con otros, se encontró que, por ejemplo, para el sexo en nuestro estudio no hubo significancia estadística de acuerdo con el estadio de desarrollo moral mientras que en otro estudio denominado Desarrollo del juicio moral en la educación superior sí se encontraron estas diferencias, indicando que las mujeres inician la educación superior con mayor desarrollo moral post convencional que los hombres y mantienen esta ventaja hacia el final de la carrera ${ }^{19}$.

En nuestro estudio se encontró que el índice A, el cual refleja el pensamiento relativista de transición entre el estadio 4 y el 5A, aunque no es estadísticamente significativo, es mayor en los hombres que en las mujeres, mientras que el estadio 4, el 5B y el 6 son mayores en las mujeres. Esto puede ser analizado desde lo expuesto en el estudio de Pérez ${ }^{20}$, cuyos hallazgos son similares al estudio de Barba ${ }^{15}$, en el que se encontró que en el pensamiento moral post convencional correspondiente al estadio 5A, las mujeres aventajan significativamente a los varones, mientras que en los estadios más altos 5B y 6 las diferencias entre sexos no gozan de magnitud suficiente como para alcanzar la significancia estadística. De igual manera, en la escala A se obtuvieron diferencias altamente significativas entre hombres y mujeres; estas últimas aparecen como más contestatarias, relativistas e inconformistas que los varones.

En el estudio de Cueto y Mueller ${ }^{21}$, razonamiento moral en cinco niveles educativos, se encontró que las mujeres obtuvieron resultados más altos que los hombres en la etapa 4 y los hombres obtuvieron resultados más altos que las mujeres en la etapa 3 , puede ser cierto que las mujeres y los hombres razonan diferente sobre cuestiones morales como lo sostiene Guilligan ${ }^{22}$; sin embargo, las tendencias encontradas no siguen la tendencia que plantea la autora. Otros estudios señalan no encontrar diferencias en el razonamiento moral entre hombres y mujeres ${ }^{23,24}$.

\section{Limitaciones del estudio}

El propósito del estudio fue describir los niveles de desarrollo del juicio moral entre los estudiantes, sin embargo, el tamaño de la muestra no permitió alto poder como la mayoría de los estudios referenciados. 
Para facilitar la comparación con los reportes previos se utilizó la prueba ANOVA, aunque solo los estadios 4, 5B y $\mathrm{P}$ se distribuyeron normalmente. Estas limitaciones hacen que se interprete cuidadosamente las diferencias reportadas para algunos puntajes entre algunos grupos de comparación, debido a que las pruebas utilizadas son menos conservativas para la evaluación de la hipótesis planteada.

Adicionalmente, se requiere incorporar otras variables (antecedentes académicos, abordajes curriculares, experiencias de vida, etc.) para determinar su influencia en el desarrollo moral.

En cuanto a la participación de estudiantes por nivel académico, cabe destacar que en el IX semestre fue escasa debido a que gran parte de la dedicación académica está destinada al desarrollo de sus prácticas clínico-comunitario, situación que dificultó el contacto en el aula.

\section{Fortalezas}

Al realizar las comparaciones entre varios grupos se verificaron los hallazgos con pruebas post hoc como la Tukey, que permitió identificar la presencia o ausencia de diferencias entre los grupos de comparación.

Implicaciones para la práctica: el medir el desarrollo moral de los estudiantes de enfermería, permite generar cambios a nivel curricular, dado que se espera que a través de la formación y las experiencias que ocurren en ella, los individuos se vuelvan autónomos y críticos.

\section{Conclusiones}

Este estudio permitió conocer el nivel de desarrollo moral de los estudiantes de enfermería y, de manera significativa, de los de tercer semestre y menores de 20 años quienes tenían un nivel de desarrollo del estadio 5A superior a los niveles más avanzados, lo cual puede deberse al poco poder de la muestra en los demás semestres participantes en el estudio y a la influencia de los conocimientos y vivencias que se adquieren en el nivel debido a la asignatura que se tiene en el plan curricular.

También se pudo concluir que la mayoría de los participantes del estudio, se centraban en un desarrollo del juicio moral ubicado en el estadio ${ }^{4}$, que corresponde al nivel convencional caracterizado por tener un sistema social y de conciencia motivada por cumplir el propio deber aceptado y dar sostén a las leyes. Asimismo, se evidenció y se confirmó que no hay una influencia directa entre un alto nivel académico del estudiante y el crecimiento moral en general.

\section{Referencias}

1. Maldonado CE. Tensión entre bioética y biopolítica. En: Aristizaval Ch, Escobar J, Maldonado C, Vega J, Sánchez F. Horizontes de la bioética: Salud y realidad social. Academia Nal. de Medicina y Universidad El Bosque, Programa de Bioética; 2004: 27-46.

2. Potter VR. Bioética puente, bioética global y bioética profunda. Cuadernos del Programa Regional de Bioética, OPS/OMS. 1997; 7: 32.

3. García G. Hacia un ethos sanitario. En bioética y justicia sanitaria. Colección Bios y Ethos $\mathrm{N}^{\circ} 9$. Bogotá: Ediciones El Bosque, 2 ${ }^{\mathrm{a}}$ Edición, 2001. p. 101.

4. Linde A. Síntesis y valoración de la teoría sobre el desarrollo moral de Lawrence Kohlberg. Ágora-Pap Filosofía. 2010; 29(2): 31-54.

5. Paravic K. Enfermería y globalización. Cienci Enferm. 2010; 16(1): 9-15. doi: 10.4067/S071795532010000100002.

6. Arbeláez R, Corredor M, Pérez M. Concepciones sobre competencias. Bucaramanga: Ediciones Universidad Industrial de Santander CEDEDUIS; 2009.

7. Kohlberg L. De lo que es a lo que debe ser. Cómo cometer la falacia naturalista y vencerla en el estudio del desarrollo moral. Buenos Aires, Prometeo Libros; 2010.

8. Narváez D. Educación y desarrollo moral. Rep Polit Soc. 2003 ; 2(2): 39-49.

9. Rest JR. Development in judging moral issues. Estados Unidos, Ed. University of Minnesota Press; 1979.

10. Amaya I, Ibáñez L. Reforma curricular del programa de enfermería. Universidad Industrial de Santander. 2004. p. 19, 31.

11. Barba B, Romo JM. Desarrollo del juicio moral en la educación superior. Rev Mex Inv Ed. 2005; 10(24): 67-92.

12. Pérez I, Dussán M. Validación de la prueba Defining Issues Test con estudiantes de medicina en la Universidad del Rosario en Colombia. Rev Electr Inv. 2009; 11(1): 1-13.

13. Céspedes S, Gonzáles A, Aguilar M, Cortés M. Desarrollo ético-moral en estudiantes de ciencias de la comunicación. Rev Cienc UANL. 2015; 75: 81-86.

14. Bužgová R, Sikorová L. Moral judgment 
competence of nursing students in the Czech Republic. Nurse Educ Today. 2013;33(10): 12011206. doi: 10.1016/j.nedt.2012.06.016.

15. Barba B. Razonamiento moral de principios en estudiantes de secundaria y de bachillerato. Rev Mex Inv Ed. 2001;6(13): 501-523.

16. Perry CM, McIntire WG. Modes of moral judgment among early adolescents. Adolescence. 1995; 30(119): 707-715.

17. Jaramillo M. Educación religiosa y desarrollo moral. [Tesis de maestría]. Pereira, Universidad Católica Popular del Risaralda; 2010.

18. Zerpa CE. Tres teorías del desarrollo del juicio moral: Kohlberg, Rest, Lind. Implicaciones para la formación moral. Laurus Rev Edu. 2007;13 (23): 137-157.

19. Moltó Brotons J, Pérez-Delgado E, Mestre Escrivá V. La variable sexo y su efecto en el desarrollo estructural y de contenido del racionamiento sociomoral. Recerca Rev Pensament Anàlisi. 1990;15(1-2): 91-115. doi: http://dx.doi. org/10.6035/Recerca.

20. Pérez E, García R, Clemente A. La capacidad de razonamiento moral en jóvenes adolescentes (13-19 años). Rev Edu. 1994; 303: 261-279.

21. Cueto S, Mueller D. Razonamiento moral en hombres y mujeres en cinco niveles educativos. Rev Psicol. 1990; 8(1): 11-20.

22. Gilligan C. In a Different Voice: Women's Conceptions of Self and of Morality. Harvard Educational Review, 1977;47(4): 481-517. doi: /10.17763/haer.47.4.g6167429416hg510.

23. Rest J. Moral development: Advances in research and theory. New York, NY: Praeger; 1986.

24. Walker L. Sex differences in the development of moral reasoning. Child Development, Lafayette, 1984;53: 677-691. doi: 10.2307/1130121. 